\title{
Is PKS 0625-354 another variable TeV active galactic nucleus?
}

\section{Dorit Glawion $^{a, *}$ and Alicja Wierzcholska ${ }^{b, c}$ on behalf of the H.E.S.S. Collaboration} (a complete list of authors can be found at the end of the proceedings)

${ }^{a}$ Friedrich-Alexander-Universität Erlangen-Nürnberg, Erlangen Centre for Astroparticle Physics, Erwin-Rommel-Str. 1, D-91058, Erlangen, Germany

${ }^{b}$ Instytut Fizyki Jadrowej PAN, ul. Radzikowskiego 152, 31-342 Krakow, Poland

${ }^{c}$ Landessternwarte, Universität Heidelberg, Königstuhl, D-69117 Heidelberg, Germany

E-mail: dorit.glawion@fau.de, alicja.wierzcholska@ifj.edu.pl

The majority of the active galactic nuclei (AGN) detected at very-high-energies above $100 \mathrm{GeV}$ belong to the class of blazars with a small angle between the jet-axis and the line-of-sight. Only about 10 percent of the gamma-ray AGN are objects with a larger viewing angle resulting in a smaller Doppler boosting of the emission. Originally, it was believed that gamma-ray emission can only be observed from blazars and those are variable in its brightness. Instead, the last years have shown that non-blazar active galaxies also show a fascinating variability behaviour which provide important new insights into the physical processes responsible for the gamma-ray production and especially for flaring events.

Here, we report on the observation of gamma-ray variability of the active galaxy PKS 0625-354 detected with the H.E.S.S. telescopes in November 2018. The classification of PKS 0625-354 is a still matter of debate. The H.E.S.S. measurements were performed as part of a flux observing program and showed in the first night of the observation a detection of the object with $>5 \sigma$. A denser observation campaign followed for the next nine nights resulting in a decrease of the gamma-ray flux. Those observations were accompanied with Swift in the X-ray and UV/optical band allowing for the reconstruction of a multi-band broad-band spectral energy distribution. We will discuss the implications of the gamma-ray variability of the object.

$37^{\text {th }}$ International Cosmic Ray Conference (ICRC 2021)

July 12 th - 23rd, 2021

Online - Berlin, Germany

\footnotetext{
${ }^{*}$ Presenter
} 


\section{Introduction}

Out of over 80 AGN detected at $\mathrm{TeV}$ energies so far ${ }^{1}$, only a few are classified as radio galaxies or sometimes called misaligned blazars: M87 $(z=0.0044)$, Cen A $(z=0.002)$, NGC 1275 $(z=0.018)$, and very recently also 3 C $264(z=0.022)$. Furthermore, IC $310(z=0.019)$ and PKS 0625-354 $(z=0.055)$, classified as radio galaxies in the literature, appear as AGN of unknown type. Contrary to blazars, radio galaxies are observed at large angle between the line of sight and the jet axis. On one hand, this results in very weak relativistic beaming making it more difficult to detect distant objects (see Fig. 1).

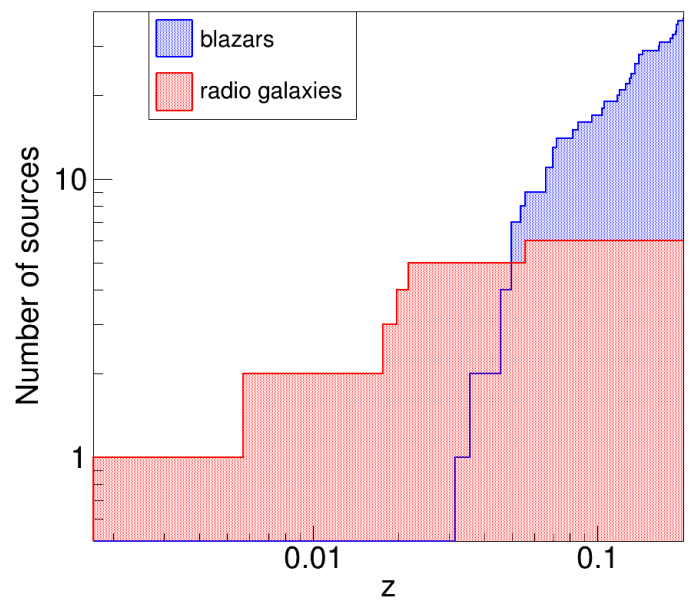

Figure 1: Cumulative number densities of active galaxies emitting TeV radiation within one gigaparsec distance to the Sun. While blazars (blue histogram) are more numerous, radio galaxies (red histogram) are the dominant population in the local universe.

However, the lack of strong beaming simplifies the interpretation and modeling of the emission of the object, as the spectral energy distribution (SED) of the source is a strong function of the often-unknown Doppler factor. Moreover, strong beaming of the jet emission in blazars might hide other emission sources, not connected with the jet, such as the magnetosphere emission or emission from the radio lobes. Therefore, such more exotic emission mechanisms are much easier to study in radio galaxies.

PKS 0625-354 is a Fanaroff-Riley (FR) I radio galaxy [1], also classified as a LINER [2]. While the optical spectrum ([OIII] line luminosity) resembling that of a BL Lac object [3], the kpc radio morphology clearly shows two extended lobe structures typical for a FR I radio galaxy. Radio observations revealed superluminal motion with $v_{\text {app }} \propto 3.0 \pm 0.5 c$ [4]. This corresponds to the limit on the observation angle of the jet $\theta \lesssim 37^{\circ}$. The jet-to-counterjet ratio of the pc-scale radio jet (one-sided) limits the viewing angle to $\theta \lesssim 57^{\circ}$. The modeling of the non-simultaneous SED with a synchrotron-self Compton model is consistent in the X-ray to gamma-ray range with the values of the observations angle of $10-19^{\circ}$ [6]. H.E.S.S. reported on the detection of PKS 0625-354 in [7]. The observed TeV gamma-ray spectral index of the object is consistent with a simple power-law of the form $\mathrm{d} N / \mathrm{d} E \propto E^{-\Gamma}$ with an index of $\Gamma=2.8 \pm 0.5$. We note that the source is affected by

1http://tevcat.uchicago.edu/ 
Extragalactic Background Light (EBL) absorption, so the intrinsic spectral index is $~ 2.5$, similar to that seen in M87 and Cen A. The gamma-ray emission of the object is rather faint. The flux measured by H.E.S.S. above $580 \mathrm{GeV}$ is $\sim 4 \%$ of Crab. However, taking into account the relatively high redshift of this radio galaxy, it is among the brightest absolute luminosities from the VHEdetected radio galaxies. No variability of the very-high-energy emission was detected by H.E.S.S., but observation lasted just 5.5 hours [7]. The average spectral index of PKS 0625-354 in 3FGL catalog is 1.89. However, a curvature is present [8]. The X-ray emission of PKS 0625-354 is dominated by the jet [6]. Observations with Suzaku show weak (10\% amplitude) X-ray variability on time scales of 1-2 days, while the Fermi-LAT observations revealed a flare in gamma-rays with a rather weak amplitude change by a factor of three [6]. The multi-wavelength light curve shows variability in the X-ray band, but no variable emission in the Optical-UV regime [7].

The search for fast variability from misaligned blazars is of particular importance because it can hardly be explained by the standard shock-in-jet model. In small emission region (as inferred from the variability time scale) $\gamma-\gamma$ pair production would lead to the absorption of the $\mathrm{TeV}$ gamma-rays making the emission impossible to be observed. This indicates highly anisotropic radiation processes (to avoid pair absorption). Possible explanations are, e.g., mini-jet structures within the jets [9], jet-cloud/star interactions where the clouds may originate from stellar winds [10], and magnetospheric models [11], similar to those known from pulsar theory.

In order to investigate the variability behaviour of PKS 0625-354 at very-high-energies, H.E.S.S. monitored the emission in November 2018. A significant signal was found in the first night of the observation with the Real-Time-Analysis of H.E.S.S. Therefore, a denser monitoring was organized together with Swift observations.

\section{H.E.S.S. observations and analysis results}

In 2018 November from MJD 58423.99 to MJD 58432.97 PKS 0625-354 was observed with H.E.S.S. in a hybrid mode with five telescopes in stereoscopic mode. The so-called "wobble mode" was used for the observation where the source position is offset by $0.7^{\circ}$ from the camera center. This allows a measurement of the background simultaneously [15]. In the first night of the observation the real-time-analysis running in parallel to the observation indicated a high significance $(\sim 5 \sigma)$ [16]. Therefore, further observations were scheduled resulting in a total of $\sim 17.5 \mathrm{~h}$ of live time of good quality data.

The data were analyzed using the ImPACT maximum likelihood-based fitting technique [17]. The background produced by cosmic-rays is rejected using a neural network based scheme. The residual background contamination level of the source region (ON and OFF) is estimated with the reflected-background method for the reconstruction of the spectrum and light curve [18]. An independent analysis chain was used for the calibration and reconstruction as a crosscheck which yield compatible result.

The analysis of $\sim 17.5 \mathrm{~h}$ of data using the ring-background method [18] results in a significance of $8.7 \sigma$. The reflected-background method resulted in $8.6 \sigma$.

The nightly-binned light curve above an energy threshold of $200 \mathrm{GeV}$ is shown in Fig. 2. Integral upper limits are given for flux data points with less than $2 \sigma$ and where calculated following [19] at the $95 \%$ confidence level. Fitting the light curve with a constant fit function yields a $\chi^{2}$ of 


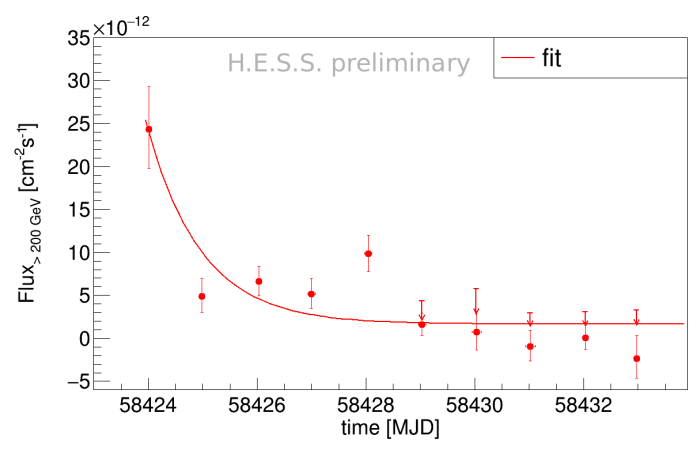

Figure 2: Preliminary nightly binned light curve above $200 \mathrm{GeV}$ measured with the H.E.S.S. telescopes in November 2018.

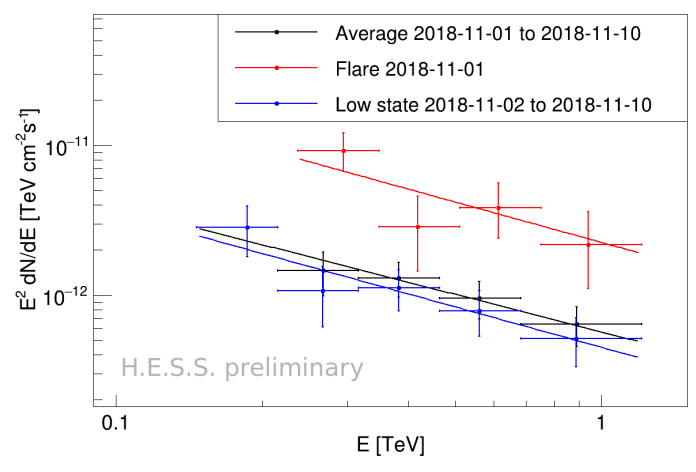

Figure 3: Preliminary spectral energy distributions of different emission states.

55 and 9 degree of freedom (d.o.f.) corresponding to a probability of $1 \times 10^{-8}$. Furthermore, we fit the light curve with the following function:

$$
F=F_{0}+F_{1} \cdot 2^{-\left|t-t_{1}\right| / \tau}
$$

where $\tau$ is the flux doubling time scale. The fit yields a $\tau=(17 \pm 1) \mathrm{h}$ with a probability of $0.02 \%$.

We performed a spectral analysis with a simple power law fit $\mathrm{d} N / \mathrm{d} E=f_{0} \cdot E^{-\Gamma}$ of all the data (average state), of only the first night (flaring state) and the remaining data (low state). The results are shown in Fig. 3 and Table 1.

\begin{tabular}{llccccc}
\hline $\begin{array}{l}\text { Emission } \\
\text { state }\end{array}$ & $\begin{array}{l}\text { dates } \\
{[\mathrm{h}]}\end{array}$ & $\begin{array}{c}f_{0} \times 10^{-12} \\
{\left[\mathrm{TeV}^{-1} \mathrm{~s}^{-1} \mathrm{~cm}^{-2}\right]}\end{array}$ & $\begin{array}{c}E_{0} \\
{[\mathrm{TeV}]}\end{array}$ & $\Gamma$ & $\begin{array}{c}\text { Energy range } \\
{[\mathrm{TeV}]}\end{array}$ & \begin{tabular}{l} 
Notes \\
\hline Average
\end{tabular} 2018-01-01-2018-01-10 \\
$7.51 \pm 1.02_{\text {stat }}$ & 0.40 & $2.83 \pm 0.26_{\text {stat }}$ & $0.15-1.20$ & \\
Flare & $2018-01-01$ & $23.8 \pm 4.9_{\text {stat }}$ & 0.44 & $2.90 \pm 0.49_{\text {stat }}$ & $0.24-1.20$ & \\
Low state & $2018-01-02-2018-01-10$ & $6.27 \pm 1.02_{\text {stat }}$ & 0.40 & $2.90 \pm 0.31_{\text {stat }}$ & $0.15-1.20$ & \\
2012 & $2012-11-2012-12$ & $0.58 \pm 0.22_{\text {stat }}$ & 1.0 & $2.84 \pm 0.50_{\text {stat }}$ & $0.2-10.0$ & {$[7]$} \\
\hline
\end{tabular}

Table 1: Preliminary results of the spectral analysis of the H.E.S.S. data. Dates are given for the night before sunset. 


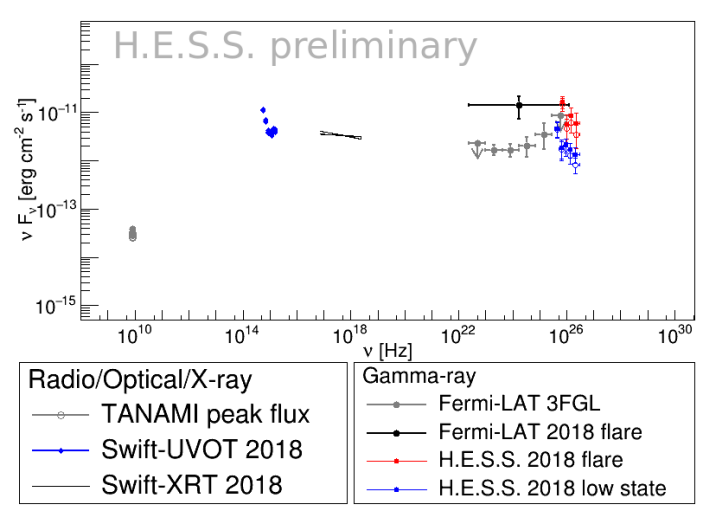

Figure 4: Preliminary multi-wavelength spectral energy distribution.

\section{Multi-wavelength spectral energy distribution}

The H.E.S.S. spectral energy distributions corrected for EBL absorption using the model from [20] are shown in the multi-wavelength SED in Fig. 4.

The SED as includes contemporaneous optical/UV and X-ray data obtained with the SwiftUVOT and XRT. The X-ray spectrum shown in the figure is the average spectrum obtained from three individual pointings on 2018-11-03, 2018-11-04, and 2018-11-05 showing a simple absorbed power-law with an energy flux density of $F_{2-10 \mathrm{keV}}=(5.03 \pm 0.20) \times 10^{-12} \mathrm{erg} \mathrm{cm}^{-2} \mathrm{~s}^{-1}$ and a photon spectral index of $2.20 \pm 0.06$. In the MeV-GeV band we show the 3FGL catalog spectrum as well as a differential flux point between $100 \mathrm{MeV}-500 \mathrm{GeV}$ measured with Fermi-LAT around the flaring episode from 2018-10-30 to 2018-11-03 with a test statistics of $T S=11$. Note that previously, only a non-simultaneous SED was investigated in [7]. Furthermore, we included radio very-long-baseline-interferometry measurements obtained with the TANAMI array [12].

\section{Implications on viewing angle}

Taking into account the flux-doubling time scale obtained from the TeV light curve we can estimate the size of the emission region.

$$
R<\delta \cdot c \cdot \tau_{\mathrm{var}} \cdot \frac{1}{(1+z)}
$$

with $z=0.055$ and $\tau_{\text {var }}=17 \mathrm{~h}$, we obtain

$$
R<\delta \cdot 1.7 \times 10^{15} \mathrm{~cm}
$$

We further compare the variability time scale with the light crossing time of the black hole assuming the mass of PKS $0625-354 M_{\mathrm{BH}}=(1.55 \pm 0.66) \times 10^{9} M_{\odot}$ as found by [14]

$$
t_{\mathrm{G}}=\frac{G M_{\mathrm{BH}}}{c^{3}}=82 \mathrm{~min} .
$$

Thus, the size of the $\mathrm{TeV}$ emission region obtained with the variability time scale is much larger than the radius of black hole assuming that it is maximally rotating. 


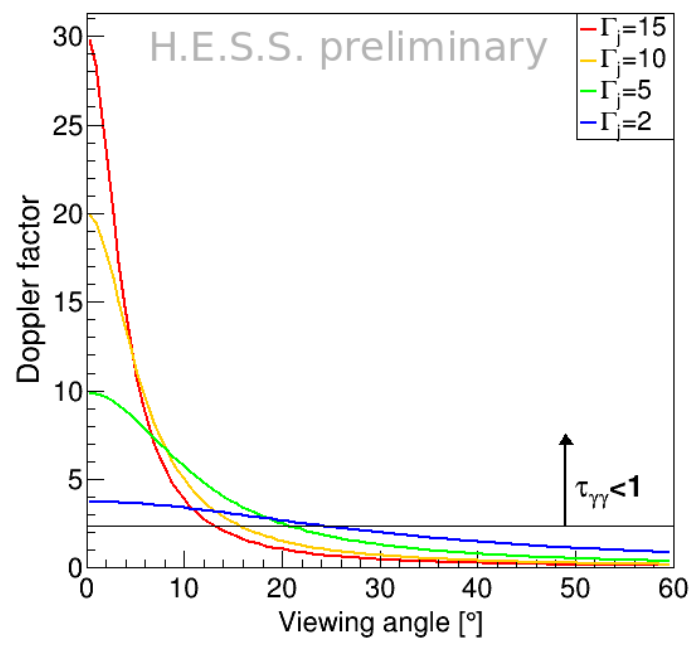

Figure 5: Doppler factor versus viewing angle as a function of the Lorentz factor. The solid black line indicates the doppler factor for which the optical depth for $\gamma \gamma$-pair-production becomes 1 .

Taking into account the small size of the $\mathrm{TeV}$ emission region we further calculate the optical depth for gamma-gamma pair production for $1 \mathrm{TeV}$ photons using Eq. 9 in [13]:

$$
\tau_{\gamma \gamma} \sim \frac{\sigma_{\mathrm{T}} D_{\mathrm{L}}^{2} F_{0} \epsilon_{\gamma}(1+z)}{10 R m_{e}^{2} c^{5} \delta^{5}}<1
$$

where $\sigma_{\mathrm{T}}$ is the Thomson cross section, $D_{\mathrm{L}}$ is the luminosity distance, $F_{0}$ the peak of the target spectral energy distribution, $\epsilon_{\gamma}$ the energy of the highest gamma-ray photon (here $1 \mathrm{TeV}$ ), $R$ is the size of the emitting region, $m_{e}$ the mass ofthe electron and $c$ the speed of light. Assuming $F_{\mathrm{K} \text {,Hbands }}=1.75 \times 10^{-11} \mathrm{erg} \mathrm{cm}^{-2} \mathrm{~s}^{-1}$ for $F_{0}$ where the absorption with the $\mathrm{TeV}$ photons is supposed to happen, this yields a constraint for the Doppler factor of $\delta>2.4$.

In Fig 5 we plot the Doppler factor $\delta=\left(\Gamma_{b}(1-\beta \cos \theta)\right)^{-1}$ as a function of the viewing angle $\theta$ assuming different bulk Lorentz factors. In addition we plot the constraint for the Doppler factor of $\delta>2.4$.

Thus, the Doppler factor constraint from the $\mathrm{TeV}$ variability provides an upper limit on the viewing angle of PKS 0625-354 of $\theta<24^{\circ}$ in comparison to the upper limit of $\theta<53^{\circ}$, reported previously based on the flux of the jet and the counterjet at parsec-scale radio maps [12].

\section{Summary and Conclusion}

For the first time we have observed variability in the very-high-energy band from the AGN PKS 0625-354. Furthermore, we presented a contemporaneous measured multi-wavelength SED of the AGN PKS 0625-354. The variability provides a possibility to get an estimate of the upper limit on the viewing angle of the object of $\theta<24^{\circ}$ which is a tighter constraint than limits from radio measurements. In the future, we will work further on the implications of the viewing angle on the multi-wavelength SED. 


\section{ACKNOWLEDGEMENTS}

H.E.S.S. gratefully acknowledges financial support from the agencies and organizations listed at https://www . mpi-hd.mpg.de/hfm/HESS/pages/publications/auxiliary/HESS-Acknowledgements-201 html. A.W. is supported by the National Science Center through the research project UMO2016/22/M/ST9/00583.

\section{References}

[1] Ojha et al., 2010, TANAMI: tracking active galactic nuclei with austral milliarcsecond interferometry. I. First-epoch $8.4 \mathrm{GHz}$ images, A\&A, 519, A45

[2] Lewis et al., 2003, Emission-Line Diagnostics of the Central Engines of Weak-Line Radio Galaxies, ApJ, 593, 115

[3] Wills et al., 2004, Emission lines and optical continuum in low-luminosity radio galaxies, MNRAS, 347, 771

[4] Müller et al., 2013, The TANAMI Program: Southern-Hemisphere AGN on (Sub-)parsec Scales, arXiv:1301.4384, Conference Proceedings of "11th European VLBI Network Symposium \& Users Meeting"

[5] Aleksić et al. (MAGIC Collaboration), 2014, Black hole lightning due to particle acceleration at subhorizon scales, Science 346, 1080

[6] Fukazawa et al., 2015, Suzaku Observations of gamma-Ray Bright Radio Galaxies: Origin of the X-ray Emission and Broad-Band Modeling, ApJ, 798, 74

[7] H.E.S.S. Collaboration et al, 2018, H.E.S.S. discovery of very high energy -ray emission from PKS 0625-354, MNRAS, 476, 4187

[8] Acero et al. (Fermi-LAT Collaboration), 2015, Fermi Large Area Telescope Third Source Catalog, ApJS, 218, 23

[9] Giannios et al. 2010, Fast TeV variability from misaligned minijets in the jet of M87, MNRAS 402,1649

[10] Barkov et al. 2012, Interpretation of the Flares of M87 at TeV Energies in the Cloud-Jet Interaction Scenario, ApJ 755, 170

[11] Levinson \& Rieger 2011, Variable TeV Emission as a Manifestation of Jet Formation in M87?, ApJ 730, 123

[12] Angioni et al. 2019, Gamma-ray emission in radio galaxies under the VLBI scope. I. Parsecscale jet kinematics and high-energy properties of $\gamma$-ray-detected TANAMI radio galaxies, A\&A, 627, A148

[13] Abdo et al. 2011, Insights into the High-energy $\gamma$-ray Emission of Markarian 501 from Extensive Multifrequency Observations in the Fermi Era ApJ, 727, 129 
[14] Bettoni et al. 2003, The black hole mass of low redshift radiogalaxies, A\&A, 399, 869

[15] Berge et al. 2007, Background modelling in very-high-energy $\gamma$-ray astronomy, A\&A, 466, 1219

[16] Balzer et al. 2014, The H.E.S.S. central data acquisition system, Astroparticle Physics, 54C, 67

[17] Parsons \& Hinton 2014, A Monte Carlo Template based analysis for Air-Cherenkov Arrays, $\mathrm{APh}, 56,26$

[18] Aharonian et al. 2006, Observations of the Crab nebula with HESS, A\&A,457, 899

[19] Rolke et al. 2005, Limits and confidence intervals in the presence of nuisance parameters NIMPA, 551, 493

[20] Dominguez et al. 2011, Extragalactic background light inferred from AEGIS galaxy-SED-type fractions MNRAS, 410, 2556 


\section{Full Authors List: H.E.S.S. Collaboration}

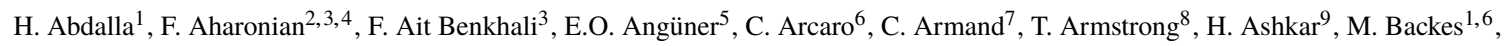
V. Baghmanyan ${ }^{10}$, V. Barbosa Martins ${ }^{11}$, A. Barnacka ${ }^{12}$, M. Barnard ${ }^{6}$, R. Batzofin ${ }^{13}$, Y. Becherini ${ }^{14}$, D. Berge ${ }^{11}$, K. Bernlöhr ${ }^{3}$, B. Bi ${ }^{15}$, M. Böttcher ${ }^{6}$, C. Boisson ${ }^{16}$, J. Bolmont ${ }^{17}$, M. de Bony de Lavergne ${ }^{7}$, M. Breuhaus ${ }^{3}$, R. Brose ${ }^{2}$, F. Brun ${ }^{9}$, T. Bulik ${ }^{18}$, T. Bylund ${ }^{14}$, F. Cangemi ${ }^{17}$, S. Caroff ${ }^{17}$, S. Casanova ${ }^{10}$, J. Catalano ${ }^{19}$, P. Chambery ${ }^{20}$, T. Chand $^{6}$, A. Chen ${ }^{13}$, G. Cotter ${ }^{8}$, M. Curyło ${ }^{18}$, H. Dalgleish ${ }^{1}$, J. Damascene Mbarubucyeye ${ }^{11}$, I.D. Davids ${ }^{1}$, J. Davies ${ }^{8}$, J. Devin ${ }^{20}$, A. Djannati-Atai ${ }^{21}$, A. Dmytriiev ${ }^{16}$, A. Donath ${ }^{3}$, V. Doroshenko ${ }^{15}$, L. Dreyer ${ }^{6}$, L. Du Plessis ${ }^{6}$, C. Duffy ${ }^{22}$, K. Egberts ${ }^{23}$, S. Einecke ${ }^{24}$, J.-P. Ernenwein ${ }^{5}$, S. Fegan ${ }^{25}$, K. Feijen ${ }^{24}$, A. Fiasson ${ }^{7}$, G. Fichet de Clairfontaine ${ }^{16}$, G. Fontaine ${ }^{25}$, F. Lott ${ }^{1}$, M. Füßling ${ }^{11}$, S. Funk ${ }^{19}$, S. Gabici ${ }^{21}$, Y.A. Gallant ${ }^{26}$, G. Giavitto ${ }^{11}$, L. Giunti ${ }^{21,9}$, D. Glawion ${ }^{19}$, J.F. Glicenstein ${ }^{9}$, M.-H. Grondin ${ }^{20}$, S. Hattingh ${ }^{6}$, M. Haupt ${ }^{11}$, G. Hermann ${ }^{3}$, J.A. Hinton ${ }^{3}$, W. Hofmann ${ }^{3}$, C. Hoischen ${ }^{23}$, T. L. Holch ${ }^{11}$, M. Holler ${ }^{27}$, D. Horns ${ }^{28}$, Zhiqiu Huang ${ }^{3}$, D. Huber ${ }^{27}$, M. Hörbe ${ }^{8}$, M. Jamrozy ${ }^{12}$, F. Jankowsky ${ }^{29}$, V. Joshi ${ }^{19}$, I. Jung-Richardt ${ }^{19}$, E. Kasai ${ }^{1}$, K. Katarzyński ${ }^{30}$, U. Katz ${ }^{19}$, D. Khangulyann ${ }^{31}$, B. Khélifi' ${ }^{21}$, S. Klepser ${ }^{11}$, W. Kluźniak ${ }^{32}$, Nu. Komin ${ }^{13}$, R. Konno ${ }^{11}$, K. Kosack ${ }^{9}$, D. Kostunin ${ }^{11}$, M. Kreter ${ }^{6}$, G. Kukec Mezek ${ }^{14}$, A. Kundu ${ }^{6}$, G. Lamanna ${ }^{7}$, S. Le Stum ${ }^{5}$, A. Lemière ${ }^{21}$, M. Lemoine-Goumard ${ }^{20}$, J.-P. Lenain ${ }^{17}$, F. Leuschner ${ }^{15}$, C. Levy ${ }^{17}$, T. Lohse ${ }^{33}$, A. Luashvili ${ }^{16}$, I. Lypova ${ }^{29}$, J. Mackey ${ }^{2}$, J. Majumdar ${ }^{11}$, D. Malyshev ${ }^{15}$, D. Malyshev ${ }^{19}$, V. Marandon ${ }^{3}$, P. Marchegiani ${ }^{13}$, A. Marcowith ${ }^{26}$, A. Mares ${ }^{20}$, G. Martí-Devesa ${ }^{27}$, R. $\operatorname{Marx}^{29}$, G. Maurin ${ }^{7}$, P.J. Meintjes ${ }^{34}$, M. Meyer ${ }^{19}$, A. Mitchell ${ }^{3}$, R. Moderski ${ }^{32}$, L. Mohrmann ${ }^{19}$, A. Montanari ${ }^{9}$, C. Moore ${ }^{22}$, P. Morris ${ }^{8}$, E. Moulin ${ }^{9}$, J. Muller ${ }^{25}$, T. Murach ${ }^{11}$, K. Nakashima ${ }^{19}$, M. de Naurois ${ }^{25}$, A. Nayerhoda ${ }^{10}$, H. Ndiyavala ${ }^{6}$, J. Niemiec $^{10}$, A. Priyana Noel ${ }^{12}$, P. O’Brien ${ }^{22}$, L. Oberholzer ${ }^{6}$, S. $\mathrm{Ohm}^{11}$, L. Olivera-Nieto ${ }^{3}$, E. de Ona Wilhelmi ${ }^{11}$, M. Ostrowski ${ }^{12}$, S. Panny ${ }^{27}$, M. Panter ${ }^{3}$, R.D. Parsons ${ }^{33}$, G. Peron ${ }^{3}$, S. Pita ${ }^{21}$, V. Poireau ${ }^{7}$, D.A. Prokhorov ${ }^{35}$, H. Prokoph ${ }^{11}$, G. Pühlhofer ${ }^{15}$, M. Punch ${ }^{21,14}$, A. Quirrenbach ${ }^{29}$, P. Reichherzer ${ }^{9}$, A. Reimer ${ }^{27}$, O. Reimer ${ }^{27}$, Q. Remy ${ }^{3}$, M. Renaud ${ }^{26}$, B. Reville ${ }^{3}$, F. Rieger ${ }^{3}$, C. Romoli ${ }^{3}$, G. Rowell ${ }^{24}$, B. Rudak ${ }^{32}$, H. Rueda Ricarte ${ }^{9}$, E. Ruiz-Velasco ${ }^{3}$, V. Sahakian ${ }^{36}$, S. Sailer ${ }^{3}$, H. Salzmann ${ }^{15}$, D.A. Sanchez ${ }^{7}$ A. Santangelo ${ }^{15}$, M. Sasaki ${ }^{19}$, J. Schäfer ${ }^{19}$, H.M. Schutte ${ }^{6}$, U. Schwanke ${ }^{33}$, F. Schüssler ${ }^{9}$, M. Senniappan ${ }^{14}$, A.S. Seyffert ${ }^{6}$, J.N.S. Shapopi ${ }^{1}$, K. Shiningayamwe ${ }^{1}$, R. Simoni ${ }^{35}$, A. Sinha ${ }^{26}$, H. Sol ${ }^{16}$, H. Spackman ${ }^{8}$, A. Specovius ${ }^{19}$, S. Spencer ${ }^{8}$, M. Spir-Jacob ${ }^{21}$, L. Stawarz ${ }^{12}$, R. Steenkamp ${ }^{1}$, C. Stegmann ${ }^{23,11}$, S. Steinmass ${ }^{3}$, C. Steppa ${ }^{23}$, L. Sun ${ }^{35}$, T. Takahashi ${ }^{31}$, T. Tanaka ${ }^{31}$, T. Tavernier ${ }^{9}$, A.M. Taylor ${ }^{11}$, R. Terrier ${ }^{21}$, J. H.E. Thiersen ${ }^{6}$, C. Thorpe-Morgan ${ }^{15}$, M. Tluczykont ${ }^{28}$, L. Tomankova ${ }^{19}$, M. Tsirou ${ }^{3}$, N. Tsuji ${ }^{31}$, R. Tuffs ${ }^{3}$, Y. Uchiyama ${ }^{31}$, D.J. van der Walt ${ }^{6}$, C. van Eldik ${ }^{19}$, C. van Rensburg ${ }^{1}$, B. van Soelen $^{34}$, G. Vasileiadis ${ }^{26}$, J. Veh ${ }^{19}$, C. Venter ${ }^{6}$, P. Vincent ${ }^{17}$, J. Vink ${ }^{35}$, H.J. Völk ${ }^{3}$, S.J. Wagner ${ }^{29}$, J. Watson ${ }^{8}$, F. Werner ${ }^{3}$, R. White ${ }^{3}$, A. Wierzcholska ${ }^{10}$, Yu Wun Wong ${ }^{19}$, H. Yassin ${ }^{6}$, A. Yusafzai ${ }^{19}$, M. Zacharias ${ }^{16}$, R. Zanin ${ }^{3}$, D. Zargaryan ${ }^{2,4}$, A.A. Zdziarski ${ }^{32}$, A. Zech ${ }^{16}$, S.J. Zhu ${ }^{11}$, A. Zmija ${ }^{19}$, S. Zouari ${ }^{21}$ and N. Żywucka ${ }^{6}$.

${ }^{1}$ University of Namibia, Department of Physics, Private Bag 13301, Windhoek 10005, Namibia

${ }^{2}$ Dublin Institute for Advanced Studies, 31 Fitzwilliam Place, Dublin 2, Ireland

${ }^{3}$ Max-Planck-Institut für Kernphysik, P.O. Box 103980, D 69029 Heidelberg, Germany

${ }^{4}$ High Energy Astrophysics Laboratory, RAU, 123 Hovsep Emin St Yerevan 0051, Armenia

${ }^{5}$ Aix Marseille Université, CNRS/IN2P3, CPPM, Marseille, France

${ }^{6}$ Centre for Space Research, North-West University, Potchefstroom 2520, South Africa

${ }^{7}$ Laboratoire d'Annecy de Physique des Particules, Univ. Grenoble Alpes, Univ. Savoie Mont Blanc, CNRS, LAPP, 74000 Annecy, France

${ }^{8}$ University of Oxford, Department of Physics, Denys Wilkinson Building, Keble Road, Oxford OX1 3RH, UK

${ }^{9}$ IRFU, CEA, Université Paris-Saclay, F-91191 Gif-sur-Yvette, France

${ }^{10}$ Instytut Fizyki Jạdrowej PAN, ul. Radzikowskiego 152, 31-342 Kraków, Poland

${ }^{11}$ DESY, D-15738 Zeuthen, Germany

${ }^{12}$ Obserwatorium Astronomiczne, Uniwersytet Jagielloński, ul. Orla 171, 30-244 Kraków, Poland

${ }^{13}$ School of Physics, University of the Witwatersrand, 1 Jan Smuts Avenue, Braamfontein, Johannesburg, 2050 South Africa

${ }^{14}$ Department of Physics and Electrical Engineering, Linnaeus University, 35195 Växjö, Sweden

${ }^{15}$ Institut für Astronomie und Astrophysik, Universität Tübingen, Sand 1, D 72076 Tübingen, Germany

${ }^{16}$ Laboratoire Univers et Théories, Observatoire de Paris, Université PSL, CNRS, Université de Paris, 92190 Meudon, France

${ }^{17}$ Sorbonne Université, Université Paris Diderot, Sorbonne Paris Cité, CNRS/IN2P3, Laboratoire de Physique Nucléaire et de Hautes Energies, LPNHE, 4 Place Jussieu, F-75252 Paris, France

${ }^{18}$ Astronomical Observatory, The University of Warsaw, Al. Ujazdowskie 4, 00-478 Warsaw, Poland

${ }^{19}$ Friedrich-Alexander-Universität Erlangen-Nürnberg, Erlangen Centre for Astroparticle Physics, Erwin-Rommel-Str. 1, D 91058 Erlangen, Germany

${ }^{20}$ Université Bordeaux, CNRS/IN2P3, Centre d'Études Nucléaires de Bordeaux Gradignan, 33175 Gradignan, France

${ }^{21}$ Université de Paris, CNRS, Astroparticule et Cosmologie, F-75013 Paris, France

${ }^{22}$ Department of Physics and Astronomy, The University of Leicester, University Road, Leicester, LE1 7RH, United Kingdom

${ }^{23}$ Institut für Physik und Astronomie, Universität Potsdam, Karl-Liebknecht-Strasse 24/25, D 14476 Potsdam, Germany

${ }^{24}$ School of Physical Sciences, University of Adelaide, Adelaide 5005, Australia

${ }^{25}$ Laboratoire Leprince-Ringuet, École Polytechnique, CNRS, Institut Polytechnique de Paris, F-91128 Palaiseau, France

${ }^{26}$ Laboratoire Univers et Particules de Montpellier, Université Montpellier, CNRS/IN2P3, CC 72, Place Eugène Bataillon, F-34095 Montpellier Cedex 5, France

${ }^{27}$ Institut für Astro- und Teilchenphysik, Leopold-Franzens-Universität Innsbruck, A-6020 Innsbruck, Austria

${ }^{28}$ Universität Hamburg, Institut für Experimentalphysik, Luruper Chaussee 149, D 22761 Hamburg, Germany 
${ }^{29}$ Landessternwarte, Universität Heidelberg, Königstuhl, D 69117 Heidelberg, Germany

${ }^{30}$ Institute of Astronomy, Faculty of Physics, Astronomy and Informatics, Nicolaus Copernicus University, Grudziadzka 5, 87-100 Torun, Poland

${ }^{31}$ Department of Physics, Rikkyo University, 3-34-1 Nishi-Ikebukuro, Toshima-ku, Tokyo 171-8501, Japan

${ }^{32}$ Nicolaus Copernicus Astronomical Center, Polish Academy of Sciences, ul. Bartycka 18, 00-716 Warsaw, Poland

${ }^{33}$ Institut für Physik, Humboldt-Universität zu Berlin, Newtonstr. 15, D 12489 Berlin, Germany

${ }^{34}$ Department of Physics, University of the Free State, PO Box 339, Bloemfontein 9300, South Africa

${ }^{35}$ GRAPPA, Anton Pannekoek Institute for Astronomy, University of Amsterdam, Science Park 904, 1098 XH Amsterdam, The Netherlands

${ }^{36}$ Yerevan Physics Institute, 2 Alikhanian Brothers St., 375036 Yerevan, Armenia 